\title{
Introduction to Intuitionistic L-fuzzy Semi Filter (ILFSF) of Lattices
}

\author{
A. Maheswari, Member, IACSIT and M. Palanivelrajan
}

\begin{abstract}
We develop a construction of an intuitionistic L-fuzzy semi filter by extending the concept of L-fuzzy semi filter based on Intuitionistic L-fuzzy Lattices. In this paper we establish some fundamental properties of Intuitionistic L-fuzzy semi filter.
\end{abstract}

Index Terms-Fuzzy subset, L-fuzzy subset, Intuitionistic L-fuzzy subset, Intuitionistic L-fuzzy semi filter.

\section{INTRODUCTION}

In 1965, Lofti A. Zadeh [5] introduced the notion of a fuzzy subset of a set as a method for representing uncertainty in real physical world. The concept of intuitionistic fuzzy set was introduced by Atanassov.K.T [1] as a generalization of the notion of fuzzy set. Palaniappan.N and et.al [4] defined the Intuitionistic L-fuzzy ideal and the homomorphism of Intuitionistic L-fuzzy ideal and established some results related to that. Due to the importance of semi filters in the classical representation theory, the main purpose of this paper is to study the generalization of these concepts for intuitionistic L-fuzzy sets. The main motivation in this work is to introduce the concept of Intuitionistic L-fuzzy semi filter and prove some results on these.

\section{PRELIMINARIES}

In this section, some well-known definitions are recalled. It will be necessary in order to understand the new concepts introduced in this paper

Definition 2.1

A fuzzy set $\mathrm{A}$ in $\mathrm{X}$ is defined as the set of ordered pairs $A=\left\{\left(x, \mu_{\mathrm{A}}(x)\right) / x \in X\right\}$, where $\mu_{\mathrm{A}}: X \rightarrow[0,1]$ is the membership function.

\section{Definition 2.2}

Let $X$ be a non-empty set $L=(L, \leq, \wedge, \vee)$ be a lattice with least element 0 and greatest element 1 . An L-fuzzy subset $A$ of $X$ is a function $\mathrm{A}: X \rightarrow L$

\section{Definition 2.3}

An Intuitionistic fuzzy set (IFS) $\mathrm{A}$ in a non-empty set $X$ is defined as an object of the form

$$
A=\left\{\left\langle x, \mu_{A}(x), \gamma_{A}(x)\right\rangle / x \in X\right\} \text { where } \mu_{A}: X \rightarrow[0,1] \text { is }
$$

Manuscript received August 9, revised September 7, 2012

The authors are with the Velammal College of Engineering and Technology, Madurai, India (e-mail:mahes_sathya@yahoo.com; palanivelrajan1975@gmail.com) the degree membership and $\gamma_{A}: X \rightarrow[0,1]$ is the degree of non-membership of the element $x \in X$ satisfying $0 \leq$ $\mu_{A}(x)+\gamma_{A}(x) \leq 1$.

Definition 2.4

A function $N: L \rightarrow L$ is an involutive order reverse operation if $N[N(a)]=a$ and $a \leq b$ iff $N(b) \leq N(a)$

\section{Definition 2.5}

Let $(L, \leq)$ be a complete lattice with least element 0 and greatest element 1 and an involutive order reversing operation $N: L \rightarrow L$. Then an Intuitionistic L-fuzzy subset (ILFS) $\mathrm{A}$ in a non-empty set $X$ is defined as an object of the form $A=\left\{\left\langle x, \mu_{A}(x), \gamma_{A}(x)\right\rangle / x \in X\right\}$ where $\mu_{A}: X \rightarrow L$ is the degree membership and $\gamma_{A}: X \rightarrow L$ is the degree of non-membership of the element $x \in X$ satisfying $\mu_{A}(x) \leq N\left(\gamma_{A}(x)\right)$.

\section{Definition 2.6}

A subset $(S, \leq)$ of a poset $(P, \leq)$ is said to be semi - filter if for $x \in S, y \in P, x \leq y$ implies $y \in S$.

\section{Definition 2.7}

An $\mathrm{L}$ - fuzzy semi filter on $P$ is a function $\mu: P \rightarrow L$ such that for $x, y \in P, x \leq y \Rightarrow \mu(x) \leq \mu(y)$.

\section{Definition 2.8}

Let $X$ and $X$ ' be any two sets. Let $\mathrm{f}: X \rightarrow X^{\prime}$ be any function and let A be IFS in X, V is IFS in $f(X)=X$, defined by $\mu_{V}(y)=\operatorname{Sup} \mu_{A}(x)$ and $\gamma_{V}(y)=\operatorname{Inf} \gamma_{A}(x)$

$$
x \in f^{-1}(y) \quad x \in f^{-1}(y)
$$

for all $x \in X$ and $y \in X^{\prime}$. A is called a pre image of $\mathrm{V}$ under $\mathrm{f}$ and is denoted by $f^{1}(V)$

\section{INTUITIONISTIC L-FUZZY SEMI FILTER}

\section{Definition 3.1}

An Intuitionistic L-fuzzy set of a Lattice is called as an Intuitionistic L-fuzzy semi filter whenever $x \leq y$, we have $\mu_{A}(x) \leq \mu_{A}(y)$ and $\gamma_{A}(x) \geq \gamma_{A}(y)$

\section{Definition 3.2}

An Intuitionistic L-fuzzy set of a Lattice is called as an Intuitionistic L-fuzzy semi ideal whenever $x \leq y$, we have $\mu_{A}(x) \geq \mu_{A}(y)$ and $\gamma_{A}(x) \leq \gamma_{A}(y)$

\section{Definition 3.3}

Let $L_{1}$ and $L_{2}$ be any two Lattices. Then the function 
f: $L_{1} \rightarrow L_{2}$ is said to be a homomorphism if
a) $f(x \vee y)=f(x) \vee f(y)$
b) $f(x \wedge y)=f(x) \wedge f(y)$

\section{Definition 3.4}

For every Intuitionistic Fuzzy set A we define $C(A)=\{<x, K, L)>/ x \in X\}$

where $K=\max _{x \in X} \mu_{A}(x)$ and $L=\min _{x \in X} \gamma_{A}(x)$

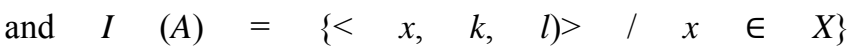
where $\mathrm{k}=\min _{x \in X} \gamma_{A}(x)$ and $l=\max _{x \in X} \mu_{A}(x)$

\section{Theorem 3.1}

The union of two Intuitionistic L-fuzzy semi filters is an Intuitionistic L-fuzzy semi filter.

Proof: Let $A$ and $B$ be two Intuitionistic L-fuzzy semi filters that is $A=\left\{\left\langle x, \mu_{A}(x), \gamma_{A}(x)\right\rangle / x \in X\right\}$ and if $x \leq y$, we have $\mu_{A}(x) \leq \mu_{A}(y)$ and $\gamma_{A}(x) \geq \gamma_{A}(y)$

$$
B=\left\{\left\langle x, \mu_{B}(x), \gamma_{B}(x)\right\rangle / x \in X\right\} \text { and if } x \leq y,
$$

we have $\mu_{B}(x) \leq \mu_{B}(y)$ and $v_{\mathrm{B}}(x) \geq v_{\mathrm{B}}(y)$

Now

$$
\begin{aligned}
& A \cup B=\left\{<\mathrm{x}, \mu_{A}(x) \vee \mu_{B}(x), \gamma_{A}(x) \wedge \gamma_{B}(x)>/ x \in X\right\} \\
& \text { TO prove: } \mu_{A}(x) \vee \mu_{B}(x) \leq \mu_{A}(y) \vee \mu_{B}(y)
\end{aligned}
$$

We know that $\mu_{A}(x) \vee \mu_{B}(x)=\sup \left\{\mu_{A}(x), \mu_{B}(x)\right\}$

If $\sup \left\{\mu_{A}(x), \mu_{B}(x)\right\}=\mu_{A}(x)$ then $\mu_{A}(x) \leq \sup$ $\left\{\mu_{A}(y), \mu_{B}(y)\right\}$

Since $\sup \left\{\mu_{A}(y), \mu_{B}(y)\right\}=\mu_{A}(y)$ then $\left.\mu_{A}(x)\right) \leq$ $\mu_{A}(y)$ and if $\sup \left\{\mu_{A}(y), \mu_{B}(y)\right\}=\mu_{B}(y)$ then $\mu_{A}(x) \leq \mu_{A}(y) \leq \mu_{B}(y) \Rightarrow \mu_{A}(x) \leq \mu_{A}(y)$.

Also if $\sup \left\{\mu_{A}(x), \mu_{B}(x)\right\}=\mu_{B}(x)$ then also $\mu_{\mathrm{B}}(\mathrm{x}) \leq$ $\sup \left\{\mu_{A}(y), \mu_{B}(y)\right\}$.

Thus $\mu_{A}(x) \vee \mu_{B}(x) \leq \mu_{A}(y) \vee \mu_{B}(y)$.

Similarly one can prove that

$$
\gamma_{A}(x) \wedge \gamma_{B}(x) \geq \gamma_{A}(y) \wedge \gamma_{B}(y)
$$

Hence the union of two Intuitionistic L-fuzzy semi filters is an Intuitionistic L-fuzzy semi filter.

\section{Theorem 3.2}

The intersection of two Intuitionistic L-fuzzy semi filters is an Intuitionistic L-fuzzy semi filter if one is contained in the other.

Proof: Let A and B be two Intuitionistic L-fuzzy semi filters.

That is $A=\left\{\left\langle x, \mu_{A}(x), \gamma_{A}(x)\right\rangle / x \in X\right\}$ and if $x \leq y$, we have $\mu_{A}(x) \leq \mu_{A}(y)$ and $\gamma_{A}(x) \geq \gamma_{A}(y)$

$$
B=\left\{\left\langle x, \mu_{B}(x), \gamma_{B}(x)\right\rangle / x \in X\right\} \text { and if } x \leq y,
$$

we have $\mu_{B}(x) \leq \mu_{B}(y)$ and $\gamma_{B}(x) \geq \gamma_{B}(y)$ $A \cap B=\left\{<\mathrm{x}, \mu_{A}(x) \wedge \mu_{B}(x), \gamma_{A}(x) \vee \gamma_{B}(x)>/ x \in X\right\}$ Let $A \subseteq B$ then $\left.\mu_{A}(x) \leq \mu_{B}(x)\right)$ and $\gamma_{A}(x) \geq \gamma_{B}(x)$.
If $B \subseteq A$ then $\mu_{B}(x) \leq \mu_{A}(x)$ and $\gamma_{B}(x) \geq v_{A}(x)$

Now $\mu_{B}(x) \leq \inf \left\{\mu_{A}(y), \mu_{B}(y)\right\}$ for $\mu_{B}(x) \leq \mu_{B}(y)$ and $\mu_{B}(x) \leq \mu_{A}(x) \leq \mu_{A}(y) \Rightarrow \mu_{B}(x) \leq \mu_{A}(y)$

Thus $\mu_{A}(x) \wedge \mu_{B}(x) \leq \mu_{A}(y) \wedge \mu_{B}(y)$

Similarly one can prove that

$$
\gamma_{A}(x) \vee \gamma_{B}(x) \geq \gamma_{A}(y) \vee \gamma_{B}(y)
$$

Hence the intersection of two Intuitionistic L-fuzzy semi filters is an Intuitionistic L-fuzzy semi filter.

\section{Theorem 3.3}

If $X$ is a lattice, then the union of a family of all intuitionistic L-fuzzy semi filters of $X$ is a intuitionistic L-fuzzy semi filter

Proof: Let $A=\{A i / i \in I\}$ be a family of ILFSF of a lattice $X$, then

$$
A=\left\{<x, \sup \left[\mu_{A i}(x)\right], \text { inf }\left[\gamma_{A i}(x)\right]>/ x \in X\right\}
$$

Let $x \leq y$

Clearly sup $\left[\mu_{A i}(x)\right] \leq \sup \left[\mu_{A i}(y)\right]$ for $\sup \left[\mu_{A 1}(x), \mu_{A 2}(x), \ldots ..\right] \sup \left[\mu_{A 1}(y), \mu_{A 2}(y), \ldots.\right]$ Also inf $\left[\gamma_{A i}(x)\right] \geq \inf \left[\gamma_{A i}(y)\right]$ for $\inf \left[v_{A 1}(x), v_{A 2}(x), \ldots.\right] \geq \inf \left[v_{A 1}(y), v_{A 2}(y), \ldots ..\right]$ Thus whenever $x \leq y$, sup $\left[\mu_{A i}(x)\right] \leq \sup \left[\mu_{A i}(y)\right]$ and $\inf \left[v_{A 1}(x), v_{A 2}(x), \ldots.\right] \geq \inf \left[v_{A 1}(y), v_{A 2}(y), \ldots ..\right]$ Hence $\mathrm{A}=\bigcup_{i \in I} A_{i}$ is an intuitionistic L-fuzzy semi filter

\section{Theorem 3.4}

The homomorphic image of an intuitionistic L-fuzzy semi filter (ILFSF) of a lattice $L_{1}$ is an intuitionistic L-fuzzy semi filter (ILFSF) of a lattice $L_{2}$

proof: Let $L_{1}$ and L2 be any two lattices.

Let f: $L_{1} \rightarrow L_{2}$ be a homomorphism.

Then (i) $f(x \vee y)=f(x) \vee f(y)$

$$
\text { (ii) } f(x \wedge y)=f(x) \wedge f(y)
$$

Let $A$ be an ILFSF of $X$

That is, if $x \leq y$, we have $\mu_{A}(x) \leq \mu_{A}(y)$ and $\gamma_{A}(x) \geq \gamma_{A}(y)$

Let $B=f(A)$

then $B=\left\{<f(x), \mu_{B}(f(x)), \gamma_{B}(f(x))>/ f(x) \in L_{2}{ }^{\prime}\right\}$

TO PROVE: $\mathrm{B}$ is an ILFSF

That is, if $f(x) \leq f(y)$ then $\mu_{B}(f(x)) \leq \mu_{B}(f(y))$ and

$\gamma_{B}(f(x)) \geq \gamma_{B}(f(y))$ clearly $B$ is an ILFSF of $L_{2}$

Let $f(x) \leq f(y)$

$$
\begin{aligned}
\mu_{B}(f(x)) \vee \mu_{B}(f(y)) & =\mu_{A}(x) \vee \mu_{A}(y) \\
& \quad\left[\text { since } \mu_{A}(x)=\mu_{B}(f(x))\right] \\
= & \mu_{A}(y)[\text { since } A \text { is an ILFSF }] \\
= & \mu_{B}(f(y))
\end{aligned}
$$

Thus $\mu_{B}(f(x)) \leq \mu_{B}(f(y))$

Also

$\gamma_{B}(f(x)) \wedge v_{B}(f(y))=\gamma_{A}(x) \wedge \gamma_{A}(y)$ 


$$
\begin{aligned}
& {\left[\text { since } \gamma_{A}(x)=\gamma_{B}(f(x))\right] } \\
= & \gamma_{A}(y) \\
= & \gamma_{B}(f(y))
\end{aligned}
$$

Therefore $\mu_{B}(f(x)) \geq \gamma_{B}(f(y))$

Thus if

$f(x) \leq f(y)$ then $\mu_{B}(f(x)) \leq \mu_{B}(f(y))$ and

$$
\gamma_{B}(f(x)) \geq \gamma_{B}(f(y))
$$

Hence $B$ is an ILFSF of $L_{2}$

\section{Theorem 3.5}

The complement of an Intuitionistic L-fuzzy semi filter is an Intuitionistic L-fuzzy semi ideal

proof: Let $A=\left\{\left\langle x, \mu_{A}(x), \gamma_{A}(x)\right\rangle / x \in X\right\}$ be an Intuitionistic L-fuzzy semi filter that is and if $\mathrm{x} \leq \mathrm{y}$, we have $\mu_{A}(x) \leq \mu_{A}(y)$ and

$$
\gamma_{A}(x) \geq \gamma_{A}(y)
$$

Now the complement of $\mathrm{A}$ is defined by

$$
\bar{A}=\left\{<\mathrm{x}, \gamma_{A}(x), \mu_{A}(x)>/ x \in X\right\}
$$

Here $\mu_{\bar{A}}(x)=\gamma_{A}(x)$ and $\gamma_{\bar{A}}(x)=\mu_{A}(x)$

From 1 it is clear that

$$
\gamma_{\bar{A}}(x) \leq \gamma_{\bar{A}}(y) \text { and } \mu_{\bar{A}}(x) \geq \mu_{\bar{A}}(y)
$$

Thus if $x \leq y$

we have $\gamma_{\bar{A}}(x) \leq \gamma_{\bar{A}}(y)$ and $\mu_{\bar{A}}(x) \geq \mu_{\bar{A}}(y)$

Therefore $\bar{A}$ is and Intuitionistic L-fuzzy semi ideal

\section{Theorem 3.6}

If $\mathrm{A}$ is an Intuitionistic L-fuzzy semi filter then $C(A)$ and I(A) are also Intuitionistic L-fuzzy semi filters

proof: Let $A=\left\{\left\langle x, \mu_{A}(x), \gamma_{A}(x)\right\rangle / x \in X\right\}$ be an Intuitionistic L-fuzzy semi filter that is and if $x \leq y$, we have

$$
\mu_{A}(x) \leq \mu_{A}(y) \text { and } \gamma_{A}(x) \geq \gamma_{A}(y)
$$

Consider

$$
C(A)=\{<x, K, L)>/ x \in X\}
$$

$$
\begin{aligned}
& \text { where } K=\max _{x \in X} \mu_{A}(x) \text { and } L=\min _{x \in X} \gamma_{A}(x) \\
& \text { and } I(A)=\{<x, k, l)>/ x \in X\} \\
& \text { where } k=\min _{x \in X} \gamma_{A}(x) \text { and } l=\max _{x \in X} \mu_{A}(x)
\end{aligned}
$$

Let $x, y \in C(A)$ and $x \leq y$

From the definition of $C(A)$, all members of $C(A)$ have the same membership degree $K$

Thus if $x \leq y, \mu_{A}(x)=\mu_{A}(y)=K$

Similarly $x \leq y$ implies $\gamma_{A}(x)=\gamma_{A}(y)=L$

Here we have the case of equality

Hence $C(A)$ is an ILFSF

Similarly one can prove that $I(A)$ is also an ILFSF

\section{ACKNOWLEDGMENT}

The authors appreciatively acknowledge the financial support of the Chairman, Velammal Educational Trust, and also acknowledge The Principal, HOD, Joint supervisor and family for their support in doing research work.

\section{REFERENCES}

[1] K. T. Atanassov, "Intuitionistic Fuzzy sets," Fuzzy Sets and Systems vol.20, no. 1, pp. $87-96,1986$.

[2] A. Kog and E. Balkanay, " $\theta$-Euclidean L-fuzzy ideals of rings," Turkish journal of mathematics, vol. 26, pp. 149 - 158, 2002.

[3] Mohammed M. Atallah, "On the L-fuzzy prime ideal theorem of distributive lattices," The journal of fuzzy mathematics, vo.1 9, no. 4, 2001.

[4] N. Palaniappan, M. Palanivelrajan, and K. Arjunan, "On Intuitionistic L-fuzzy ideal of Lattices,” Antarctica J. Math. vol.5, no. 2, pp. 53-57, 2008.

[5] L. A. Zadeh, "Fuzzy sets," Inform.Control. vol.8, pps.338-353, 1965.

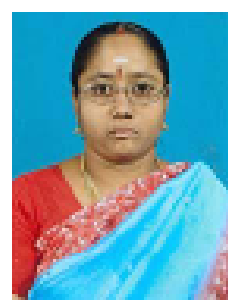

Mrs. A. Maheswari is a research scholar of Anna University of Technology, Madurai in the area of Fuzzy Algebra. Mrs. A. Maheswari born on $15^{\text {th }}$ March 1971 in Dindigul, Tamil nadu (India). Mrs. A. Maheswari completed her Bachelor Degree B.Sc., mathematics in the year of 1991, \& Master Degree M.Sc., M.Phil., mathematics in 1993 and 1999 respectively through Madurai Kamaraj University, Madurai, India. Now she is a registered scholar of Anna University of Technology, Madurai.

She is a member of IACSIT. She is having 12 years of teaching experience in various institutions. Now she is working as an assistant professor in Velammal College of Engineering \& Technology, Madurai, Tamil Nadu, India. This is her first paper published by the author. Her current research deals with fuzzy algebra. 